\title{
Minimally Invasive Reconstruction of Anterior Talofibular Ligament with Partial Peroneus Brevis Tendon in the Treatment of Chronic Lateral Ankle Instability
}

\author{
${ }^{1}$ Qian-bo Chen, ${ }^{2}$ Xiao-kang Tan, ${ }^{3}$ Chen-song Yuan, ${ }^{4}$ Xu Tao, ${ }^{5}$ Hong-hui Cao, ${ }^{6}$ Jian-zhong Xu, ${ }^{7}$ Kang-lai Tang
}

\begin{abstract}
Background: Chronic lateral ankle instability causes significant problems in physical activity and accelerates development of osteoarthritic changes. Many procedures were designed to reconstruct the anterior talo-fibular ligament (ATFL) in the treatment of chronic lateral ankle instability. Although most of them were effective, but brought big trauma and sacrifice of some tendons.
\end{abstract}

Objective: To design a minimally invasive ATFL reconstruction with partial peroneus brevis tendon and evaluate its clinical outcomes.

Study design: Nonrandomized controlled clinical trial.

Materials and methods: From 2004 to 2012, 29 patients of chronic lateral ankle instability were treated with minimally invasive ATFL reconstruction with partial peroneus brevis tendon. A $3 \mathrm{~cm}$ curved incision was made to explore the ATFL origin and its insertion. Half peroneus brevis tendon was taken to reconstruct the ATFL through the bone tunnel from the insertion of CFL to the insertion of ATFL in the fibular, and then fixed to ATFL insertion location on the talus. All patients were followed-up by radiology and clinical examination at least two years. Their ATFLs were always evaluated by standard stress $X$-ray examination and magnetic resonance imaging (MRI) prior to surgery and every 1 year after the operation. Functional results were assessed in terms of Karlsson score and the American Orthopaedic Foot and Ankle Society (AOFAS) anklehind foot score.

Results: The average follow-up period was 57.9 months (24114 months). The majority of results $(93.1 \%)$ were satisfactory. The mean Karlsson score improved from 41.7 prior to surgery to 88.6 and AOFAS from an average 47.2 preoperatively to 91.7 postoperatively at the final follow-up visit. Paired t-tests showed improvements of great significance $(p<0.01)$. The ligaments were proved be reconstructed well in all patients by MRI. It showed the negative talar tilt sign postoperatively by stress $X$-rays. There was no recurrence of lateral ankle instability.

Conclusion: The minimally invasive ATFL reconstruction with partial peroneus brevis tendon has advantages of small trauma, good reconstruction and excellent clinical outcomes, thus, is a

\footnotetext{
${ }^{1-5}$ Orthopedic Surgeon, ${ }^{6,7}$ Professor and Head

${ }^{1-7}$ Department of Orthopedic Surgery, Southwest Hospital Third Military Medical University, Chongqing, China
}

Corresponding Author: Kang-lai Tang, Department of Orthopedic Surgery, Southwest Hospital, Third Military, Medical University, Gaotanyan Str. 30, Shapingba District, Chongqing400038, China, Phone/Fax: 0086-2365656500, e-mail: tangkanglai@hotmail.com safe and effective method for the treatment of chronic lateral ankle instability.

Keywords: Ankle instability, Reconstruction, Lateral collateral ligament, Chronic instability.

How to cite this article: Chen Q, Tan X, Yuan C, Tao X, Cao $\mathrm{H}, \mathrm{Xu} J$, Tang K. Minimally Invasive Reconstruction of Anterior Talofibular Ligament with Partial Peroneus Brevis Tendon in the Treatment of Chronic Lateral Ankle Instability. J Foot Ankle Surg (Asia-Pacific) 2014;1(1):12-16.

Source of support: Nil

Conflict of interest: None

\section{INTRODUCTION}

Chronic ankle joint lateral instability after a severe sprain or repetitive injury is a very common pathology in the active population. Approximately, 15 to $20 \%$ of acute ankle sprain change to chronic ankle joint lateral instability, which need surgical intervention. ${ }^{1}$ The anterior talofibular ligament (ATFL) and calcaneofibular (CFL) ligaments are the primary stabilizers of the lateral side of the ankle. The ATFL is the most commonly torn. ${ }^{2}$ To our knowledge, more than 80 surgical techniques have been described for reconstructing ankle ligaments and correcting ankle instability, most with 80 to $90 \%$ success rate. ${ }^{3,4}$ We present our own modification of technique, minimally invasive reconstruction of ATFL with partial peroneus brevis tendon.

\section{MATERIALS AND METHODS}

\section{Patients}

Minimally invasive reconstruction of ATFL was performed during the period 2004-2012, 29 patients (19 male, 10 female; 12 right, 17 left), with a mean age of 26.1 years (range 16-55 years). The average follow-up period was 57.9 months (range 24-114 months). At surgery, all patients were isolated ATFL tears, combination of both ATFL and CFL tears was excluded in the study. The PTF ligament was intact in all patients. In this group, 9 were professional athletes, including 5 football players, 3 basketball players and 1 table tennis player. The diagnosis was confirmed by their history, physical examination and radiological evaluation, including standard stress X-ray examination and MRI. All patients had a history of either recurrent ankle sprains or a chronic post-traumatic ankle instability combined with pain. There 
were 24 cases with edema on the anteroinferior aspect of the lateral ankle; 27 cases with palpation pain at the talar and fibular attachment of ATFL. Twenty-five had a positive anterior drawer sign and 14 had a positive talar tilt sign.

\section{Surgical Technique}

Patients were placed in the supine position with a sand bag under the affected limb and received nerve-blocking anesthesia before the operation. A tourniquet was used on the upper leg (pressure of $280 \mathrm{~mm} \mathrm{Hg}$ ). A $3 \mathrm{~cm}$ curved incision was made on the anteroinferior aspect of the lateral ankle to expose lateral talus facet, the ATFL, its origin on the talus, its insertion on the fibular, the apex of the fibula, the part of peroenus brevis tendon above inferior extensor retinaculum, the insertion of CFL on the fibular by 'moving windows' technique (Fig. 1).

After pulling and identifying the peroneus brevis tendon, another $1 \mathrm{~cm}$ vertical incision was made about $6 \mathrm{~cm}$ above the tip of the lateral malleolus to explore and split the peroneus brevis tendon longitudinally (Fig. 2). The half tendon was liberated distally to inferior extensor retinaculum (Fig. 3), and measured its length and diameter. The superior and inferior extensor retinaculum was protected during this procedure.

The bone tunnel was made from the insertion of CFL to the insertion of ATFL in the fibular, which size depended on the diameter of the liberated tendon. The freed tendon passed through the drill hole from posterior to anterior and the tendon stump was fixed on the talus by suture-anchor or bone tunnel with bridge (Fig. 4).

\section{Postoperative Management and Assessment}

The affected ankle was immobilized at a $90^{\circ}$ angle with a short leg plaster for 6 weeks with static functional exercise postoperatively. The patients began to walk with partial weight bearing through full plaster immediately after surgery, and restored sports training after 90 days.



Fig. 1: A $3 \mathrm{~cm}$ curved incision is made on the anteroinferior aspect of the lateral ankle

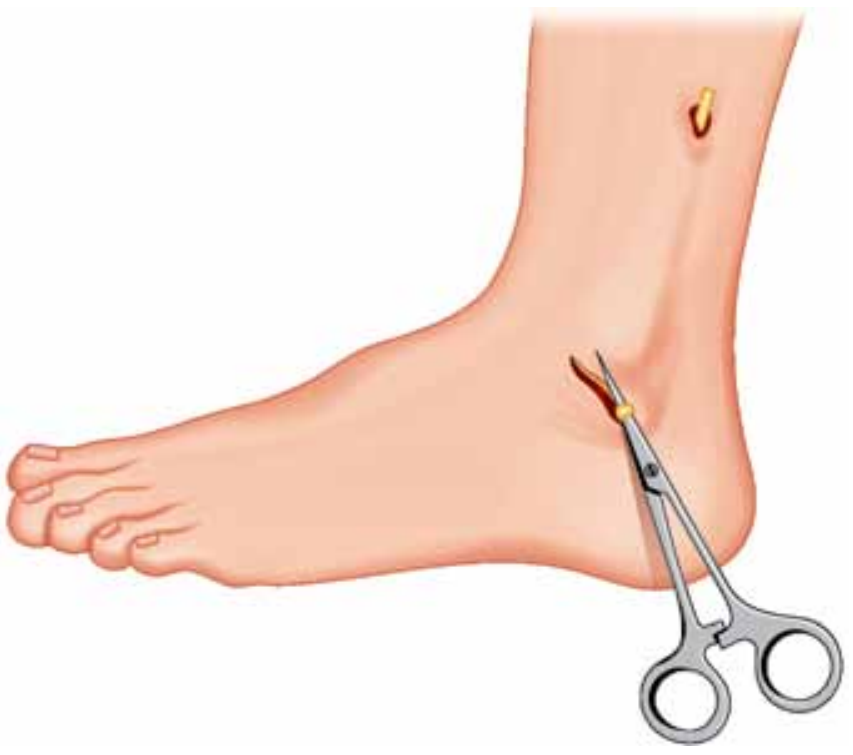

Fig. 2: Another $1 \mathrm{~cm}$ vertical incision is made about $6 \mathrm{~cm}$ above the tip of the lateral malleolus and finding peroneus brevis tendon once again

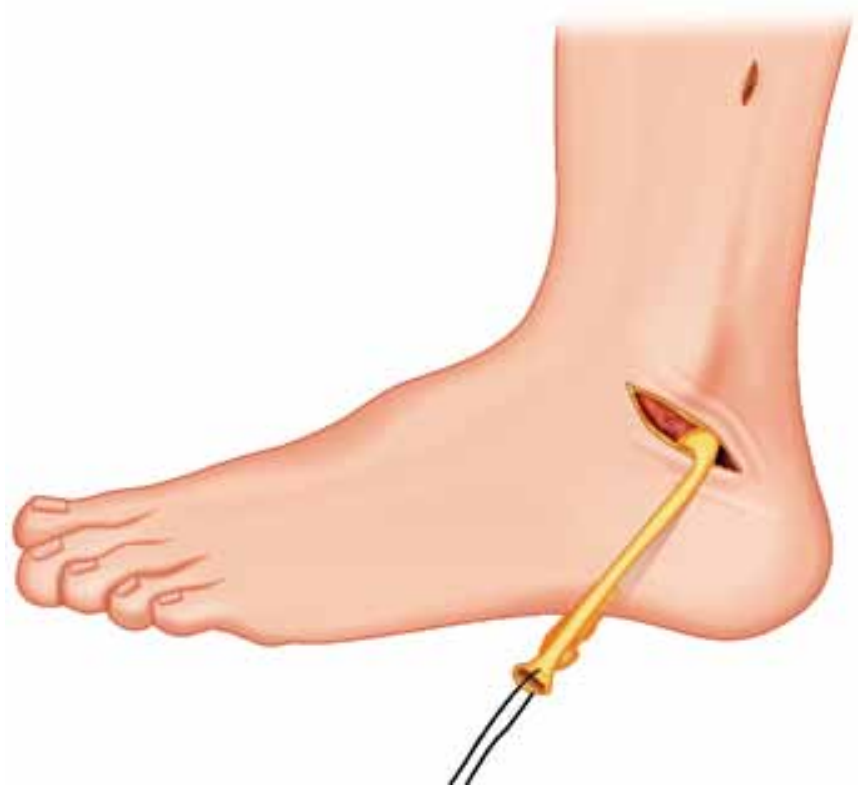

Fig. 3: Half of the peroneus brevis tendon is liberated distally to inferior extensor retinaculum

All patients were assessed both clinically and radiologically 6 months and every 1 year after surgery. Karlsson score and AOFAS were used to evaluate clinical results. Our clinical examination also focused on ankle mobility, and on possible laxity assessed by lateral coaptation defect amplitude measured in prone position, corresponding to the sum of talocrural and subtalar joint mobility. The radiological assessments included X-rays and MRI scans postoperatively.

\section{RESULTS}

All patients were followed-up for mean duration of 57.9 months (24-114 months) postoperatively. $93.1 \%$ patients ( 27 of 29 cases) were satisfied with their clinical 
results. One case complaint of mild pain near the operation site, which cured by local block, another case with mild restriction of inversion.

The mean Karlsson score improved from 41.7 (range 27 to 57) prior to surgery to 88.6 (range 62 to 100) and AOFAS from an average 47.2 (range 37 to 56) preoperatively to 91.7 (range 71 to 100) postoperatively at the final follow-up visit. Paired t-tests showed improvements of great significance $(\mathrm{p}<0.01)$.

When compared with the surgical findings, the MRIdemonstrated the exact extent of the ATFL injuries in all cases preoperatively. There were no false-positive findings. In each case, the postoperative MRI showed a newly-formed ligament structure at the former ATFL position (Fig. 5). Stress $\mathrm{X}$-ray test showed the negative talar tilt sign postoperatively (Figs 6A and B).

Twenty-three patients restored to normal walk and sports 6 months postoperatively, and 28 cases 1 year later. At the final follow-up, no recurrence of the instability and ankle restriction of sagittal motion (dorsiflexion and plantarflexion) were detected (Figs 7A and B), no skin necrosis and wound infection.

For the athletes in this series, all 9 athletes restored sports training 3 months postoperatively, and 7 of them returned to the same level before injury; only 2 football players had a mild restriction of inversion, but satisfied with their clinical results.

\section{DISCUSSION}

Ankle sprain plays a main role in lateral collateral ligaments injuries of the ankle. The structural and functional integrity of the ATFL and CFL is the foundation of the lateral ankle stability, and lateral ankle ligament injuries involving the ATFL and the CFL are among the most frequently encoun-



Fig. 4: The freed tendon is passed through the drill hole from posterior to anterior and the tendon stump is fixed on the talus by bone tunnel with bridge tered injuries in clinical situations. ${ }^{2,5-8}$ Thus, all procedures were designed to reconstruct or repair the lateral collateral ligaments.



Fig. 5: MRI showed that the ATFL was well-reconstructed and remodeled postoperatively

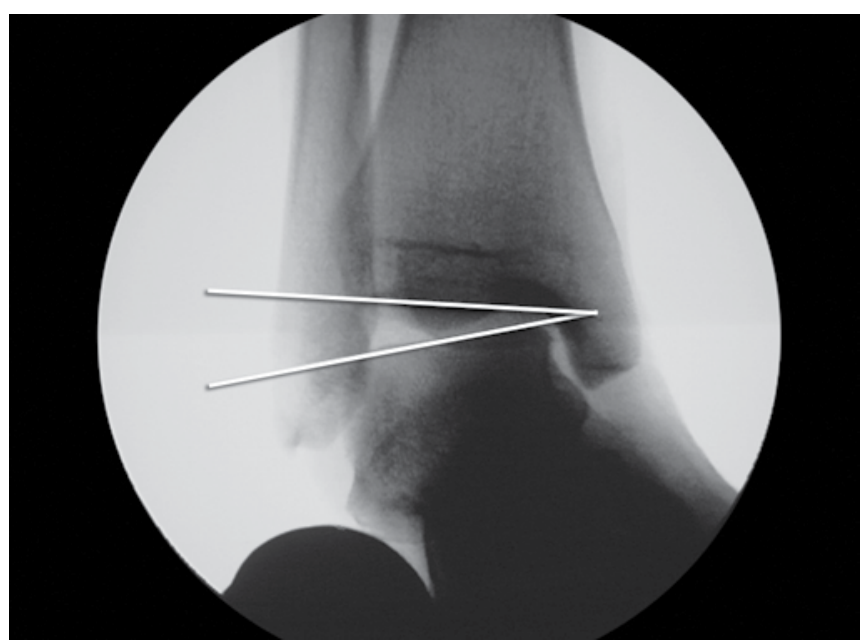

Fig. 6A: Stress X-ray showed positive talar tilt sign (talar tilt $>9^{\circ}$ ) preoperatively



Fig. 6B: Stress X-ray showed a stable ankle joint (talar tilt $<9^{\circ}$ ) postoperatively 




Fig. 7A: There was no difference in dorsiflexion between the ankle with surgery and the unaffected ankle postoperatively

Approximately, 80 kinds of surgical procedures have been reported for treatment of chronic lateral ankle instability. ${ }^{3,4}$ These techniques has been broadly divided into anatomical repair and ankle-ligament reconstruction. ${ }^{9}$ As an anatomical repair procedure, the modified Brostrom technique is a good choice for correcting lateral ankle instability. It has the advantage of improvement of the ankle stability effectively and without any complications postoperatively and the success rate was as high as $96 \% .{ }^{10}$ However, its limitations are also obvious. Girard et al thought that the modified Brostrom would eventually fail in patients who were overweight or hyperflexible or engaged in strenuous work or athletic activities in the long run. ${ }^{11}$ Barbari et al found cases in which lateral ligamentous structures were insufficient, making a repair impossible and reconstructed procedure necessary. ${ }^{12}$ Watson-Jones and Chrisman-Snook procedures are the most frequently used techniques for correcting chronic lateral ankle instability in reconstructed procedures, and Watson-Jones tendonsis has been regarded as a better method compared with other techniques. Hamido et al reported that Watson-Jones technique can improve the stability of the ankle effectively and the rate of recurrence of lateral ankle instability is low. ${ }^{13}$ However, as a non-anatomical procedure, it ruins the normal anatomical structure and mechanical function of lateral ankle. Thus, it has some disadvantages of loss of peroneal brevis muscle function, and decrease range of motion especially of the subtalar joint. In addition, its bone tunnel is difficult to drill and the incidence of freed tendon is insufficient for reconstruction is relatively high, because the freed tendon must be surrounded by three bony channels made on the tip of the fibular and talar neck in this procedure. Chrisman-Snook is regarded as another fair choice for the treatment of chronic lateral ankle instability. It reconstruct both ATFL and CFL, and can improve the stability of the lateral ankle effectively. However, according to some literatures, its wound is heavy and easy to cause skin



Fig. 7B: There was no difference in plantarflexion between the ankle with surgery and the unaffected ankle postoperatively

necrosis near the operation site. Besides, as a nonanatomical reconstructed technique, it would cause restriction of inversion of the ankle as well. ${ }^{14,15}$

The goal of lateral ankle reconstruction is to stabilize the ankle without compromising hindfoot or ankle mobility and without complications. ${ }^{9,16}$ After generalized advantages and disadvantages of some widespread used surgical procedures, we began to use a modified method of reconstruction of lateral ankle ligaments by partial peroneus brevis tendon. The technique can be briefed as liberating half of the peroneus brevis tendon to inferior extensor retinaculum distally, then, pass the freed tendon through the straight bone tunnel made on the tip of the fibula posterior to anterior and the tendon stump was fixed on the talus. All patients were followed-up for mean duration of 57.9 months postoperatively. Twenty-three patients restored to normal walk and sports 6 months postoperatively and 28 cases 1 year later. MRI showed the ligaments were reconstructed well in all patients. Stress X-ray showed the negative talar tilt sign postoperatively. The mean Karlsson score improved from 41.7 prior to surgery to 88.6 and AOFAS from an average 47.2 preoperatively to 91.7 postoperatively at the final follow-up visit. Paired t-tests showed improvements of great significance $(p<0.01)$. Two had lateral ankle pain which caused by scan healed by the treatment of local block. For the athletes in this series, we had good clinical results: seven cases returned to the same level as preoperatively and only 2 (football player) had a mild restriction of inversion. Compared with other surgical procedures, we had a satisfactory clinical result in this trial.

As some literatures reported, many previous techniques were at the cost of entire peroneus brevis tendon for reconstruction of lateral ATFL. ${ }^{4,9,13,15}$ However, peroneus brevis have a function of supporting eversion and extorsion of the ankle. Therefore, the normal function of the ankle will be affected if we cut off the tendon completely during the 
operation. To avoid the negative effect, we use half of peroneus brevis tendon for reconstruction, and the superior/inferior extensor retinaculum was protected during the procedure so as to maintain the function and structure intact of the ankle as far as possible, hence promoted the rehabilitation of ankle function. Because of the skin of foot and ankle lack of blood supply relatively, local skin necrosis caused by operation is a common problem we have to meet. Therefore, we adopt mini-open (the average length of the wound is $3.5 \mathrm{~cm}$ ) in procedure to protect local blood supply. In our study, no patient was found to have skin necrosis and joint dysfunction caused by operation and scar contracture. Anatomical reconstruction of lateral ankle ligament can keep normal function of ankle and decrease the rate of complication postoperatively as far as possible. Therefore, we keep the exit of the bone tunnel and the tendon insertion on the talus according with the start/insertion point of ATFL to the utmost in our surgery. During the follow-up period, of 29 patients, only 2 had a mild restriction of inversion and no restriction of sagittal motion (dorsiflexion and plantarflexion) were detected. As mentioned before, the bone tunnel is hard to make in Watson-Jones technique. To overcome the defection, we designed only one channel for reconstruction of ATFL. The method simplify the surgery steps, while receive a satisfactory clinical results. To summarize, the advantages of minimally invasive reconstruction of lateral collateral ligaments for the treatment of chronic lateral ankle instability using partial peroneus brevis tendon can be illustrated as follows: (1) the procedure is easy to deal with, minor operative wound protected the local blood supply and lower the incidence of local skin necrosis, (2) superior/inferior extensor retinaculum was protected during the procedure and maintain the function and structure intact of the ankle as far as possible. (3) peroneus brevis tendon was saved to the utmost and (4) anatomical reconstruction of ATFL. However, the method only reconstructed the ATFL and inability to correct combination of ATFL and CFL injury. In addition, this study was based on a small sample size.

\section{CONCLUSION}

The minimally invasive ATFL reconstruction with partial peroneus brevis tendon has advantages of small trauma, good reconstruction and excellent clinical outcomes, thus is a safe and effective method for the treatment of chronic lateral ankle instability. Nevertheless, as a modified surgical technique, further improvement of its effect evaluation and manipulation is needed.

\section{REFERENCES}

1. Molloy AP, Ajis A, Kazi H. The modified brostrom-gould procedure-early results using a newly described surgical technique. Foot Ankle Surg. Available at http://dx.doi.org/10.1016/j. fas2014.01.002

2. Buerer Y, Winkler M, Burn A, Chopra S, Crevoisier X. Evaluation of a modified brostrom-gould procedure for treatment of chronic lateral ankle instability: a retrospective study with critical analysis of outcome scoring. Foot Ankle Surg 2013;19(1):36-41.

3. Rosenbaum D, Bertsch C, Claes LE. Tenodeses do not fully restore ankle joint loading characteristics: a biomechanical in vitro investigation in the hind foot. Clin Biomech 1997;12(3): 202-209.

4. Guillo S, Bauer T, Lee JW, Takao M. Consensus in chronic ankle instability: aetiology, assessment, surgical indications and place for arthroscopy. Orthop Traumatol Surg Res 2013;99(8): S411-419.

5. Chou MC, Yeh LR, Chen CK, Pan HB, Chou YJ, Liang HL. Comparison of plain MRI and MR arthrography in the evaluation of lateral ligamentous injury of the ankle joint. J Chin Med Assoc 2006;69(1):26-31.

6. Fujii T, Luo ZP, Kitaoka HB, An KN. The manual stress test may not be sufficient to differentiate ankle ligament injuries. Clin Biomech 2000;15:619-623.

7. Tang KL. The procedure of repair damaged collateral ligament of ankle. Operative surgery of the foot and ankle (Chinese). Chongqing: Science and technology press 2006;59-62.

8. Taser F, Shafiq Q, Ebraheim NA. Anatomy of lateral ankle ligaments and their relationship to bony landmarks. Surg Radiol Anat 2006;28:391-397.

9. Baumhauer JF, O'Brien T. Surgical considerations in the treatment of ankle instability. J Athl Train 2003;37(4):458-462.

10. Fujii T, Kitaoka HB, Watanabe K, Luo ZP, An KN. Comparison of modified brostrom and evans procedures in simulated lateral ankle injury. Med Sci Sports Exer 2006;38(6):10251031.

11. Girard P, Anderson RB, Davis WH, Isear JA, Kiebzak GM. Clinical evaluation of the modified brostrom-evans procedure to restore ankle stability. Foot Ankle Int 1999;20:246-252.

12. Barbari SG, Brevig K, Egge T. Reconstruction of the lateral ligamentous structures of the ankle with a modified watson-jones procedure. Foot Ankle 1987;7(6):362-368.

13. Hamido F, Ibrahim S, Abo- El-Noor T, Al-Misfer A, Mutairi H. Salem H. Evaluation of the results of watson jones tenodesis in chronic lateral instability of the ankle. J Foot Ankle Surg 2007;13:56-62.

14. Cheng M, Tho KS. Chrisman-snook ankle ligament reconstruction outcomes-a local experience. Singapore Med J 2002;43(12): 605-609.

15. Snook GA, Chrisman OD, Wilson TC. Long-term results of the chrisman-snook operation for reconstruction of the lateral ligaments of the ankle. J Bone Joint Surg Am 1985;67(1):1-7.

16. Coughlin MJ, Schenck RC Jr, Grebing BR, Treme G. Comprehensive reconstruction of the lateral ankle for chronic instability using a free gracilis graft. Foot Ankle Int 2004;25(4): 231-241. 